Eur. Phys. J. B 10, 5-8 (1999)

\title{
Free energy of closed membrane with anisotropic inclusions
}

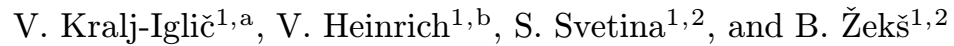 \\ 1 Institute of Biophysics, Medical Faculty, University of Ljubljana, Lipičeva 2, 1000 Ljubljana, Slovenia \\ 2 J. Stefan Institute, Jamova 39, 1000 Ljubljana, Slovenia
}

Received 3 August 1998 and Received in final form 3 May 1999

\begin{abstract}
Phospholipid membrane forming a closed surface and decorated with anisotropic inclusions is considered. The inclusions are free to redistribute laterally over the membrane and to orient in the plane of the membrane according to the local membrane curvature. A phenomenological expression for the energy of the interaction between an inclusion and local curvature of the surrounding membrane is proposed. Considering this single-inclusion energy, and assuming thermodynamic equilibrium, the free energy of the inclusions, and the consistently related lateral and orientational distributions of the inclusions are obtained using statistical mechanical methods. For a vesicle shape with given surface area, enclosed volume, and total amount of inclusions, the free energy is in general given in a nonlocal form (i.e. it can not be expressed as an integral of its area density over the membrane area). The limits of weak and strong orientational ordering are considered. Specifically, it is shown that in the shape sequence representing the formation of an exovesicle the effect of the membrane curvature on the orientation of the inclusions may stabilize the shape where the exovesicle and the cell are connected by a narrow neck.
\end{abstract}

PACS. $05.70 . \mathrm{Np}$ Interface and surface thermodynamics - 87.16.Dg Membranes, bilayers and vesicles

The system considered is composed of a continuum of phospholipid molecules into which anisotropic laterally mobile molecules are embedded. It was determined that embedding a molecule into the membrane may involve several $k T$ of energy [1]. Thus it can be expected that the embedded molecules may considerably influence the membrane free energy and also the equilibrium vesicle shape. The effect of the embedded molecules on the vesicle shape was also observed in experiments $[2,3]$.

In previous works, nonuniform lateral distribution of the embedded molecules was theoretically described by taking into account the interdependence between the local membrane curvature and the lateral density of the embedded molecules $[4,5]$, while orientation of the anisotropic embedded molecules in the local curvature field was studied [6] by considering the area density of the embedded molecules to be uniform. In this work we present a consistent description of both, the nonuniform lateral distribution and the orientational ordering of the membrane constituents.

Below we employ the term inclusion for an entity consisting of the embedded molecule and some lipids that are significantly distorted due to the presence of the embed-

\footnotetext{
a e-mail: vera.kralj-iglic@biofiz.mf.uni-lj.si

b Present address: Dept. of Biomedical Engineering, Boston University, 44 Cummington Street, Boston, MA 02215, USA
}

ded molecule. It is imagined that there exists a local membrane shape (and orientation) which fits the inclusion. We will refer to this shape as to the intrinsic shape of the inclusion. As the region where the configuration of the lipid molecules is significantly distorted is small with respect to the lateral membrane dimensions [7], the inclusions are for simplicity treated as points.

The energy of the inclusion derives from the mismatch between the local membrane shape and the intrinsic shape of the inclusion. By treating the membrane as a continuum we implicitly take into account that an inclusion of any shape behaves as a quadrupole in the curvature field. The local membrane shape is represented by curvatures of all possible normal cuts of the membrane through the site of the inclusion. For the energy of the inclusion we propose a phenomenological expression consisting of two terms,

$$
E=\frac{\xi}{4 \pi} \int_{0}^{2 \pi}\left(C-C_{\mathrm{m}}\right)^{2} \mathrm{~d} \psi+\frac{\xi^{\star}}{16 \pi} \int_{0}^{2 \pi}\left(\frac{\mathrm{d}}{\mathrm{d} \psi}\left(C-C_{\mathrm{m}}\right)\right)^{2} \mathrm{~d} \psi
$$

where $\xi$ and $\xi^{\star}$ are positive interaction constants, $C$ is the curvature of the normal cut that is for an angle $\psi$ rotated in the principal axes system of the membrane and $C_{\mathrm{m}}$ is the curvature of the normal cut corresponding to the intrinsic shape in the same direction. The first contribution takes into account the differences 
of the curvatures of the normal cuts of the two systems while the second contribution takes into account the effect of the difference between the neighbouring curvatures of the normal cuts of the two systems. The orientation of the embedded molecules is described by considering that the membrane principal directions are in general different from the principal directions of the intrinsic shape of the inclusion. The mutual orientation of the two systems is determined by the angle $\omega$. Then, using the Euler equations for the curvatures of the respective normal cuts of the continuum $C=C_{1} \cos ^{2} \psi+C_{2} \sin ^{2} \psi$ and $C_{\mathrm{m}}=C_{1 \mathrm{~m}} \cos ^{2}(\psi+\omega)+C_{2 \mathrm{~m}} \sin ^{2}(\psi+\omega)$, where $C_{1}$ and $C_{2}$ are the principal curvatures describing the local shape of the membrane and $C_{1 \mathrm{~m}}$ and $C_{2 \mathrm{~m}}$ are the principal curvatures describing the intrinsic shape of the inclusion, and performing the necessary integrations, we get

$$
\begin{aligned}
E(\omega)= & \frac{\xi}{2}\left(\bar{C}-\bar{C}_{\mathrm{m}}\right)^{2} \\
& +\frac{1}{2} \frac{\xi+\xi^{\star}}{2}\left(\hat{C}^{2}-2 \hat{C} \hat{C}_{\mathrm{m}} \cos (2 \omega)+\hat{C}_{\mathrm{m}}^{2}\right),
\end{aligned}
$$

where $\bar{C}=\frac{1}{2}\left(C_{1}+C_{2}\right), \hat{C}=\frac{1}{2}\left(C_{1}-C_{2}\right), \bar{C}_{\mathrm{m}}=\frac{1}{2}\left(C_{1 \mathrm{~m}}+\right.$ $\left.C_{2 \mathrm{~m}}\right)$ and $\hat{C}_{m}=\frac{1}{2}\left(C_{1 \mathrm{~m}}-C_{2 \mathrm{~m}}\right)$. If $\hat{C}_{\mathrm{m}}=0$ the inclusion is isotropic while if $\hat{C}_{\mathrm{m}} \neq 0$ the inclusion is anisotropic. In the previous work [5] the energy $E$ was determined by one constant. The energy $E$ is now determined by two independent constants. Analogously, the description of the continuum at a given site is also given by two independent constants [8].

To derive the free energy of the inclusions, the membrane is divided into small patches which however contain large number of molecules. The membrane curvature is taken to be constant over the patch. Statistical mechanical approach is used. The chosen patch is considered as a system of a constant area $A^{\mathrm{p}}$ and a constant number of molecules $M$ which is immersed in a heat bath so that its temperature $T$ is constant. The molecules in the patch are explicitly considered to be independent and indistinguishable. Assuming that the system is in thermodynamic equilibrium the canonical partition function of the inclusions in the small patch of the membrane is $Q=\frac{q^{M}}{M !}$, where $q$ is the partition function of an inclusion, taking into account all possible orientations of the inclusion [6]. The partition function $q$ is $q=\frac{1}{2 \pi} \int \exp \left(-\frac{E(\omega)}{k T}\right) \mathrm{d} \omega$, with $k$ the Boltzmann constant. In the partition function of the inclusion the contribution of the orientational states $q_{\text {orient }}$ is distinguished from the contribution of the other states $q_{\mathrm{c}}, q=q_{\mathrm{c}} q_{\text {orient }}$,

$$
\begin{aligned}
q_{\mathrm{c}} & =\exp \left(-\frac{\xi}{2 k T}\left(\bar{C}-\bar{C}_{\mathrm{m}}\right)^{2}-\frac{\xi+\xi^{\star}}{4 k T}\left(|\hat{C}|^{2}+\left|\hat{C}_{\mathrm{m}}\right|^{2}\right)\right) \\
q_{\text {orient }} & =\frac{1}{2 \pi} \int \exp \left(\frac{\left(\xi+\xi^{\star}\right) \hat{C} \hat{C}_{\mathrm{m}} \cos (2 \omega)}{2 k T}\right) \mathrm{d} \omega
\end{aligned}
$$

The integration in equation (4) over $\omega$ yields the modified Bessel function $\mathrm{I}_{0}[6]$ which in our case has the argument $\left(\xi+\xi^{\star}\right) \hat{C} \hat{C}_{\mathrm{m}} / 2 k T$. Knowing the canonical partition function of the patch $Q$, we obtain the Helmholtz free energy of the patch, $F^{\mathrm{p}}=-k T \ln Q$. The Stirling approximation is used and the area density of the number of molecules $m=\frac{M}{A^{\mathrm{p}}}$ is introduced. This gives for the area density of the free energy

$$
\frac{F^{\mathrm{p}}}{A^{\mathrm{p}}}=-k T m \ln \left(q_{\mathrm{c}} \mathrm{I}_{0}\left(\frac{\xi+\xi^{\star}}{2 k T} \hat{C} \hat{C}_{\mathrm{m}}\right)\right)+k T m \ln m .
$$

To obtain the free energy of the whole layer $F_{\mathrm{m}}$ the contributions of all the patches are summed, i.e., the integration over the layer area $A$ is performed $F_{\mathrm{m}}=\int \frac{F^{\mathrm{p}}}{A^{\mathrm{p}}} \mathrm{d} A$, where $\mathrm{d} A$ is the area element.

The explicit dependence of the area density $m$ on the position can be determined by the condition for the free energy of the layer to be at its minimum in the thermodynamic equilibrium of the whole system so that $\delta F_{\mathrm{m}}=0$. It is taken into account that the total number of the inclusions $M_{\mathrm{T}}$ in the layer is fixed,

$$
\int_{A} m \mathrm{~d} A=M_{\mathrm{T}} .
$$

The above isoparametric problem is reduced to the ordinary variational problem by constructing a functional $F_{\mathrm{m}}+\lambda_{\mathrm{m}} \int_{A} m \mathrm{~d} A=\int_{A} L(m) \mathrm{d} A$, where

$$
L(m)=-k T m \ln \left(q_{\mathrm{c}} \mathrm{I}_{0}\left(\frac{\xi+\xi^{\star}}{2 k T} \hat{C} \hat{C}_{\mathrm{m}}\right)\right)+k T m \ln m+\lambda_{\mathrm{m}} m
$$

and $\lambda_{\mathrm{m}}$ is the Lagrange multiplier. The variation is performed by solving the Euler equation $\frac{\partial L}{\partial m}=0$. Deriving equation (7) with respect to $m$ and taking into account equation (6) gives

$$
\frac{m}{m_{\mathrm{u}}}=\frac{q_{\mathrm{c}} \mathrm{I}_{0}\left(\frac{\xi+\xi^{\star}}{2 k T} \hat{C} \hat{C}_{\mathrm{m}}\right)}{\frac{1}{A} \int q_{\mathrm{c}} \mathrm{I}_{0}\left(\frac{\xi+\xi^{\star}}{2 k T} \hat{C} \hat{C}_{\mathrm{m}}\right) \mathrm{d} A},
$$

where $q_{\mathrm{c}}$ is given by (3) and $m_{\mathrm{u}}$ is defined by $m_{\mathrm{u}} A=M_{\mathrm{T}}$.

To obtain the equilibrium free energy of the layer the equilibrium area density (8) is inserted into the expression (5) and integrated over the area $A$. Rearranging the terms yields

$$
F_{\mathrm{m}}=-k T M_{\mathrm{T}} \ln \left(\frac{1}{A} \int q_{\mathrm{c}} \mathrm{I}_{0}\left(\frac{\xi+\xi^{\star}}{2 k T} \hat{C} \hat{C}_{\mathrm{m}}\right) \mathrm{d} A\right) .
$$

The equilibrium free energy of the inclusions (Eq. (9)) can not be generally expressed as an integral of the area density of the free energy. We say that the contribution of the inclusions is a nonlocal one. A change of the local conditions affects the cell shape and the distribution of the inclusions through the minimization of the free energy of the whole membrane. The physical origin of this effect is different from the nonlocal effect of the relative stretching of the multilayered membranes that can be attributed only to the systems consisting of two or more layers [9]. The nonlocal effect contained in equation (9) can be attributed to any closed membraneous system (including 
a monolayer). The expression for $F_{\mathrm{m}}$ obtained in [5] is recovered from equation (9) for $\hat{C}_{\mathrm{m}}=0$ and $\xi^{\star}=0$.

Further, it should be noted that although the inclusions are explicitly treated as independent, their mutual influence is taken into account through the mean curvature field which in turn depends on the lateral distribution of the inclusions.

The energy of the decorated membrane is not scale invariant (Eq. (9)) as is the case for the bending energy [10]. The inclusions favour a certain packing arrangement that depends on the absolute values of the principal membrane curvatures.

For $E / k T \ll 1$ and $C_{1}, C_{2} \ll C_{1 \mathrm{~m}}, C_{2 \mathrm{~m}}$ in all the points of the membrane the exponential function in equation (3), and the logarithmic function as well as the modified Bessel function in equation (9) can be expanded. An expansion up to the second order in the principal membrane curvatures gives the membrane free energy in terms of two invariants of the local curvature tensor, the trace and the determinant, i.e. the mean curvature $\left(C_{1}+C_{2}\right) / 2$ and the Gaussian curvature $C_{1} C_{2}$. The free energy is expressed in the form of the generalized bilayer couple model [11] with renormalized constants: the local, the nonlocal and the Gaussian bending constants, $\left(k_{\mathrm{c}}, k_{\mathrm{r}}\right.$ and $k_{\mathrm{G}}$, respectively), the spontaneous curvature $C_{0}$ and the difference between the outer and the inner membrane layer areas in the unstressed state $\triangle A_{0}$, $k_{\mathrm{c}, \mathrm{eff}}=k_{\mathrm{c}}-M_{\mathrm{T}} \xi^{2} \bar{C}_{\mathrm{m}}^{2} / 4 A k T-M_{\mathrm{T}}\left(\xi+\xi^{\star}\right)^{2} \hat{C}_{\mathrm{m}}^{2} / 32 A k T$, $k_{\mathrm{r}, \mathrm{eff}}=k_{\mathrm{r}}+M_{\mathrm{T}} \xi^{2} \bar{C}_{\mathrm{m}}^{2} / 4 A k T, k_{\mathrm{G}, \mathrm{eff}}=k_{\mathrm{G}}+M_{\mathrm{T}}(\xi+$ $\left.\xi^{\star}\right)^{2} \hat{C}_{\mathrm{m}}^{2} / 16 A k T, C_{0, \text { eff }}=\frac{k_{\mathrm{c}}}{k_{\mathrm{c}, \mathrm{eff}}}\left(C_{0}+M_{\mathrm{T}} \xi \bar{C}_{\mathrm{m}} / 2 A k_{\mathrm{c}}\right)$, $\triangle A_{0, \text { eff }}=k_{\mathrm{r}} \triangle A_{0} / k_{\mathrm{r}, \text { eff }}$. The orientational ordering of the inclusions in this case shifts the shape within the well known phase diagram of the bilayer couple model $[14,10]$. It should be noted, however, that this approximation can be used only for the shapes which nowhere exhibit large difference between the principal membrane curvatures.

For $E / k T \geq 1$ and $\hat{C}, \hat{C}_{\mathrm{m}} \neq 0$ in all the points of the membrane the limit of strong ordering may be applied. This gives for the free energy of the inclusions

$$
\begin{aligned}
& F_{\mathrm{m}}=-k T M_{\mathrm{T}} \\
& \times \ln \left(\frac{1}{A} \int \frac{\exp \left(-\frac{\xi}{2 k T}\left(\bar{C}-\bar{C}_{\mathrm{m}}\right)^{2}-\frac{\xi+\xi^{\star}}{4 k T}\left(|\hat{C}|-\left|\hat{C}_{\mathrm{m}}\right|\right)^{2}\right)}{\sqrt{\frac{\pi\left(\xi+\xi^{\star}\right)|\hat{C}|\left|\hat{C}_{\mathrm{m}}\right|}{k T}}} \mathrm{~d} A\right) .
\end{aligned}
$$

In this case the free energy of the inclusions is expressed by another set of the invariants of the local curvature tensor: the mean curvature $\bar{C}$ and the curvature deviator $|\hat{C}|$ [12]. We say that in this case the membrane exhibits deviatoric properties $[13,6]$. This approximation can be used only for the shapes which everywhere exhibit large difference between the principal membrane curvatures, as for example thin cylinders and twisted stripes.

In general, a closed shape of a fixed area and volume could have regions where $|\hat{C}|$ is very small and regions where it is large (as in the budding where the bud is connected to the main body by a narrow neck). The interdependence between the local membrane curvature and

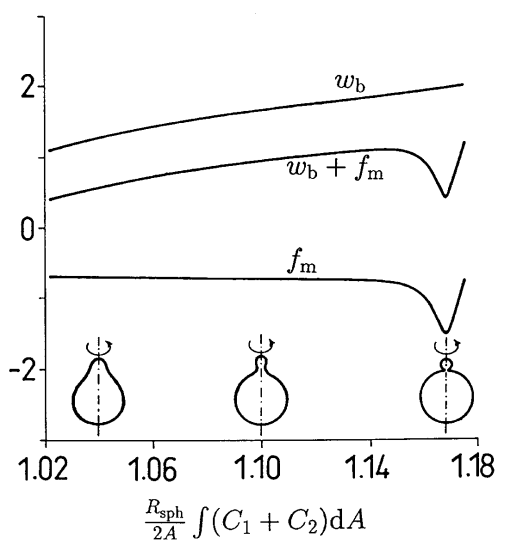

Fig. 1. The relative membrane bending energy $w_{\mathrm{b}}=$ $\frac{1}{16 \pi} \int\left(C_{1}+C_{2}\right)^{2} \mathrm{~d} A$, the relative free energy of the inclusions $f_{\mathrm{m}}=F_{\mathrm{m}} / 8 \pi k_{\mathrm{c}}$, where $k_{\mathrm{c}}$ is the membrane isotropic bending constant, and the sum of the two quantities $w_{\mathrm{b}}+f_{\mathrm{m}}$ in dependence of the relative average mean curvature of the vesicle $\frac{R_{\mathrm{sph}}}{2 A} \int\left(C_{1}+C_{2}\right) \mathrm{d} A$. The energy contributions, the average mean curvature and the principal curvatures are normalized with respect to the corresponding quantities of a sphere with the area $A[10]$. In the energy of the inclusions the constant factor is irrelevant. The parameters used in calculation are $M_{\mathrm{T}} k T / 8 \pi k_{\mathrm{c}}=1, \bar{C}_{\mathrm{m}}=0, \hat{C}_{\mathrm{m}} R_{\mathrm{sph}}=100, \xi / k T R_{\mathrm{sph}}^{2}=0.001$, $\xi^{\star}=\xi, V /\left(4 \pi R_{\mathrm{sph}}^{3} / 3\right)=0.95$ where $R_{\mathrm{sph}}=(A / 4 \pi)^{1 / 2}$.

the orientation of the anisotropic inclusions expressed by the modified Bessel function $\mathrm{I}_{0}$ enables the description to transform continuously from the limit where the orientational effects are very small and the membrane may be described as a two dimensional liquid to the regime where the inclusions are strongly ordered and the deviatoric properties of the membrane are exhibited.

Previous studies of the equilibrium vesicle shapes within a given topology $[14,10,15]$ are based on the minimization of the membrane bending energy $\frac{k_{c}}{2} \int\left(C_{1}+\right.$ $\left.C_{2}\right)^{2} \mathrm{~d} A$ at fixed membrane area $A$ and fixed enclosed volume $V$. As the form of the free energy of the inclusions in general differs from the membrane bending energy and involves also another invariant $|\hat{C}|$, the set of possible solutions of the variational problem would be different. Derivation of the new differential equations and their solution are out of scope of this work. However, it is possible to demonstrate the importance of the deviatoric effect caused by anisotropic inclusions in a following approximative manner.

We consider axisymmetrical vesicle shapes obtained by the minimization of the membrane bending energy for a sequence in which only one daughter vesicle is formed from a pear shape. Figure 1 shows the membrane bending energy, the free energy of the inclusions and the membrane free energy which is the sum of the above contributions, in dependence of increasing imposed average mean curvature. Any shape in the sequence was given by the values of the principal curvatures for a grid of points on the surface. The free energy of the inclusions was integrated numerically taking into account these values. 
For calculation of the modified Bessel function $\mathrm{I}_{0}$ we used the algorithm given in [16]. The values of the model parameters are obtained as follows. We took $M_{\mathrm{T}} k T / 8 \pi k_{\mathrm{c}}=$ 1 , where the number of inclusions $M_{\mathrm{T}}$ is of the order of $10^{3}$ and the membrane bending constant is $\simeq 10^{-19} \mathrm{~J}[12]$; to estimate the intrinsic anisotropy, we took that the inclusion favours a symmetric saddle for which $\bar{C}_{\mathrm{m}}=0$ and $\hat{C}_{\mathrm{m}}=1 / 300 \AA^{-1}[6]$ while $R_{\mathrm{sph}}=6 \mu \mathrm{m}$, corresponding to a giant phospholipid vesicle; the constant of the interaction between the inclusion and the surrounding membrane $\xi=\xi^{\star}$, can be estimated by assuming that the energy cost in distorting a tail of a phospholipid molecule within the inclusion is approximately equal to the energy difference corresponding to the tail packing in different aggregation geometries. Such difference is of the order of (0.1-0.5)kT per tail of the phospholipid molecule [17]. If we take that there are $\simeq 10$ molecules (20 tails) involved in the inclusion, the energy of the inclusion could reach several $k T$. We calculated that at the mother vesicle (towards the end of the sequence of Fig. 1), $\bar{C} R_{\mathrm{sph}} \simeq 1$ while $\hat{C} R_{\mathrm{sph}} \simeq 0$. Then, from the above choice of $\bar{C}_{\mathrm{m}}$ and $\hat{C}_{\mathrm{m}}$, and equation (2), we estimated that the interaction constant $\xi / k T R_{\mathrm{sph}}^{2}$ is of the order of $10^{-3}$.

The membrane bending energy monotonously increases along the sequence in Figure 1 [15]. The free energy of the inclusions only slightly decreases as long as the developing neck is wide. When the neck becomes narrow the free energy of the inclusions sharply decreases, reaches a minimum and then increases, as the area involved in the neck diminishes. If there are less inclusions present, if their anisotropy is weaker and if the interaction constants are smaller, the minimum is less pronounced and becomes eventually unnoticeable (not shown here). It can be deduced from the $f_{\mathrm{m}}$ curve that for smaller average mean curvature the inclusions would have a negligible effect on the shape. However, when the neck becomes narrower, the inclusions favour the neck. Therefore it is expected that the equilibrium shapes calculated by including $F_{\mathrm{m}}$ might be significantly different from the shapes at the end of the sequence. Nevertheless, the results presented in Figure 1 give insight into the effect of the deviatoric properties of the inclusions on the stability of the shapes exhibiting narrow necks and tethers.

An experimental system in which the effect of the inclusions can be significant is the erythrocyte in which the membrane skeleton-bilayer interactions are abolished, so that the erythrocyte shape is determined by the properties of the bilayer. This happens e.g. when erythrocytes are incubated at very high $\mathrm{pH}$ values. Recently, tethers connecting the parent cell and the daughter vesicle were observed in erythrocytes at these conditions [18]. It can be speculated that these tethers may be a consequence of the accumulation and orientational ordering of the anisotropic membrane embedded proteins in the tether. Moreover, cylindrical microexovesicles free of membrane skeleton were observed upon adding a cationic dimeric amphiphile to the erythrocyte suspension [3], which could be interpreted on the basis of the deviatoric properties of the membrane induced by anisotropic inclusions [19].
We are indebted to A. Iglič and A.A. Boulbitch for helpful discussions.

\section{References}

1. R. Lipowsky, Europhys. Lett. 30, 197 (1995); N. Dan, S.A. Safran, Biophys. J. 75, 1410 (1998); S. May, A. Ben-Shaul, Biophys. J. 76, 751 (1999).

2. J. Simon, M. Kühner, H. Ringsdorf, E. Sackmann, Chem. Phys. Lipids 76, 241 (1995); C.R. Safinya, Coll. Surf. A 128, 183 (1997); A. Polozova, F.M. Winnik, Biochim. Biophys. Acta 1326, 213 (1997); M. Goulian, O.N. Mesquita, D.K. Fygenson, C. Nielsen, O.S. Andersen, A. Libchaber, Biophys. J. 74, 328 (1998).

3. H. Hägerstrand, B. Isomaa, Biochim. Biophys. Acta 1109, 117 (1992)

4. V.S. Markin, Biophys. J. 36, 1 (1981); S. Leibler, J. Phys. France 47, 507 (1986); D. Andelman, T. Kawakatsu, K. Kawasaki, Europhys. Lett. 19, 57 (1992); U. Seifert, Phys. Rev. Lett. 70, 1335 (1993); A.A. Boulbitch, Phys. Rev. E 56, 3395 (1997).

5. V. Kralj-Iglič, S. Svetina, B. Žekš, Eur. Biophys. J. 24, 311 (1996).

6. J.B. Fournier, Phys. Rev. Lett. 76, 4436 (1996).

7. D.R. Fattal, A. Ben-Shaul, in Handbook of Nonmedical Applications of Liposomes, edited by D.D. Lasic, Y. Barenholz (CRC Press, Boca Raton, 1996), Vol. I, pp. 129.

8. W. Helfrich, Z. Naturförsch. 28c, 693 (1973).

9. E.A. Evans, Biophys. J. 14, 923 (1974); E.A. Evans, Biophys. J. 30, 265 (1980); S. Svetina, M. Brumen, B. Žekš, Stud. Biophys. 110, 177 (1985); S. Svetina, B. Žekš, Eur. Biophys. J. 21, 251 (1992).

10. S. Svetina, B. Žekš, Eur. Biophys. J. 17, 101 (1989).

11. V. Heinrich, S. Svetina, B. Žekš, Phys. Rev. E 48, 3112 (1993); L. Miao, U. Seifert, M. Wortis, H.G. Döbereiner, Phys. Rev. E 49, 5389 (1994).

12. E.A. Evans, R. Skalak, Mechanics and Thermodynamics of Biomembranes (CRC Press Inc., Boca Raton, 1980).

13. T.M. Fischer, J. Phys. II France 2, 337 (1992); T.M. Fischer, J. Phys. II France 3, 1795 (1993).

14. H.L. Deuling, W. Helfrich, J. Phys. France 37, 1335 (1976); K. Berndl, J. Käs, R. Lipowsky, E. Sackmann, U. Seifert, Europhys. Lett. 133, 1182 (1990); U. Seifert, K. Berndl, R. Lipowsky, Phys. Rev. A 44, 1182 (1991); J. Käs, E. Sackmann, R. Podgornik, S. Svetina, B. Žekš, J. Phys. II France 3, 631 (1993).

15. S. Svetina, B. Žekš, J. Theor. Biol. 146, 115 (1990).

16. W.H. Press, B.P. Flannery, S.A.Teukolsky, W.T. Vetterling, Numerical Recipes in Pascal (Cambridge University Press, Cambridge MA, 1989), pp. 198.

17. A. Ben-Shaul, W.M. Gelbart, in Micelles, membranes, microemulsions, and monolayers, edited by W.M. Gelbart, A. Ben-Shaul, D. Roux (Springer Verlag, New York, 1994), pp. 47.

18. M. Bobrowska-Hägerstrand, A. Iglič, H. Hägerstrand, Cell. Mol. Biol. Lett. 2, 9 (1997); V. Kralj-Iglič, A. Iglič, M. Bobrowska-Hägerstrand, H. Hägerstrand (unpublished).

19. A. Iglič, V. Kralj-Iglič, P. Peterlin, H. Hägerstrand, Cell. Mol. Biol. Lett. 3, 443 (1998). 